\title{
Scaling in the Fan of an Unconventional Quantum Critical Point
}

\author{
Roger G. Melko ${ }^{1,2}$ and Ribhu K. Kaul ${ }^{3}$ \\ ${ }^{1}$ Department of Physics and Astronomy, University of Waterloo, Ontario, N2L 3G1, Canada \\ ${ }^{2}$ Materials Science and Technology Division, Oak Ridge National Laboratory, Oak Ridge TN, 37831 \\ ${ }^{3}$ Department of Physics, Harvard University, Cambridge MA, 02138
}

(Dated: October 29, 2018)

\begin{abstract}
We present results of extensive finite-temperature Quantum Monte Carlo simulations on a SU(2) symmetric $S=1 / 2$ quantum antiferromagnet with a four-spin interaction [Sandvik, Phys. Rev. Lett. 98, $227202(2007)]$. Our simulations, which are free of the sign-problem and carried out on lattices containing in excess of $1.6 \times 10^{4}$ spins, indicate that the four-spin interaction destroys the Néel order at an unconventional $z=1$ quantum critical point, producing a valence-bond solid paramagnet. Our results are consistent with the 'deconfined quantum criticality' scenario.
\end{abstract}

Research into the possible ground states of $\mathrm{SU}(2)$ symmetric quantum antiferromagnets has thrived over the last two decades, motivated to a large extent by the undoped parent compounds of the cuprate superconductors. In these materials, the $\mathrm{Cu}$ sites can be well described as $S=1 / 2$ spins on a two-dimensional (2D) square lattice that interact with an anti-ferromagnetic exchange, the archetypal model for which is the Heisenberg model. By now, it is well established [1] that the ground state of this model with nearest-neighbor interaction has Néel order that spontaneously breaks the $\mathrm{SU}(2)$ symmetry. Two logical questions immediately arise: What possible paramagnetic ground states can be reached by tuning competing interactions that destroy the Néel state? Are there universal quantum-critical points $(\mathrm{QCP})$ that separate these paramagnets from the Néel phase?

An answer to the first question is to disorder the Néel state by the proliferation of topological defects in the Néel order parameter [2]. It was shown by Read and Sachdev [3] that the condensation of these defects in the presence of quantum Berry phases results in a fourfold degenerate paramagnetic ground state, which breaks square-lattice symmetry due to the formation of a crystal of valence bonds - a valence-bond solid (VBS) phase. An answer to the second question was posed in recent work by Senthil et al. [4], where the possibility of a direct continuous Néel-to-VBS transition was proposed. The natural field theoretic description of this 'deconfined quantum critical point' is written in terms of certain fractionalized fields that are confined on either side of the $\mathrm{QCP}$ and become 'deconfined' precisely at the critical point. As is familiar from the general study of QCPs, these fractional excitations are expected to influence the physics in a large fan-shaped region that extends above the critical point at finite- $T$ [5] (see Fig. 11).

It is clearly of great interest to find models that harbor a direct Néel-VBS QCP and that can be studied without approximation on large lattices. Currently, the best candidate is the 'JQ' model, introduced by Sandvik [6], which is an $S=1 / 2, \mathrm{SU}(2)$ invariant antiferromagnet

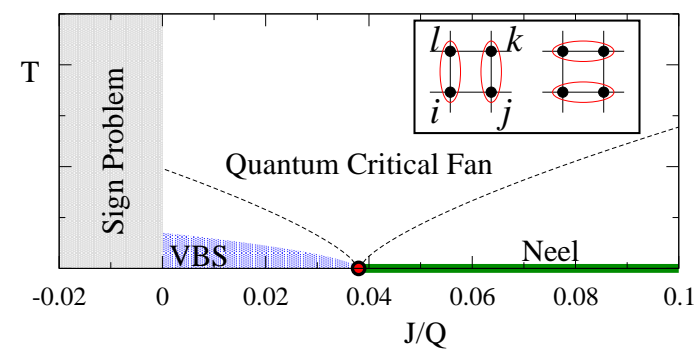

FIG. 1: (color online) Schematic of the proposed $T-J / Q$ phase diagram of the JQ model. Large-scale finite-T simulations presented here substantiate the following: (i) The $T=0$ Néel order present for $J / Q \gg 1$ is destroyed at a QCP $(J / Q \approx 0.038)$; (ii) In the 'quantum critical fan', there is scaling behavior characteristic of a $z=1 \mathrm{QCP}$; (iii) An accurate estimate of the scaling dimension of the Néel field establishes that this transition is not in the $O(3)$ universality class; and, (iv) The paramagnetic ground state for sufficiently small $J / Q$ is a VBS. In the QMC basis used here, the region with $Q<0$ is sign problematic. The inset shows how the frustrating $Q$ term is written in terms of bonds on a plaquette.

with a frustrating four-spin interaction,

$$
H_{\mathrm{JQ}}=J \sum_{\langle i j\rangle} \mathbf{S}_{i} \cdot \mathbf{S}_{j}-Q \sum_{\langle i j k l\rangle}\left(\mathbf{S}_{i} \cdot \mathbf{S}_{j}-\frac{1}{4}\right)\left(\mathbf{S}_{k} \cdot \mathbf{S}_{l}-\frac{1}{4}\right),
$$

where indices are arranged as in the inset of Fig. 1. Using a $T=0$ projector Quantum Monte Carlo (QMC) method on lattices sizes up to $32 \times 32$ [6], Sandvik showed that the four-spin interaction destroys Néel order and produces a VBS phase at $J / Q \sim 0.04$. Close to this critical value of $J / Q$, scaling in the spin and dimer correlation functions suggests a continuous transition with anomalous dimensions of the Néel and VBS order parameters equal, with a common value $\eta=0.26(3)$. In this Letter, we explore the candidate Néel-VBS QCP in the full $T-J / Q$ phase diagram on large lattices using a complementary finite- $T$ QMC technique, the Stochastic Series Expansion (SSE) method with directed loops [7]. The SSE QMC allows access to the physically important quantum critical fan (see Fig. 1), and admits high-accuracy estimates for the spin stiffness, $\rho_{S}$, and the uniform susceptibility, $\chi_{u}$. The 


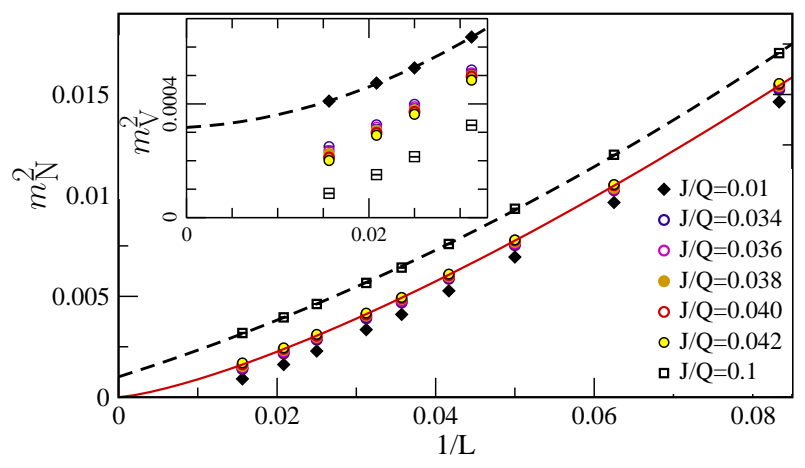

FIG. 2: (color online) $T \rightarrow 0$ converged Néel (main) and VBS (inset) order parameters as a function of $1 / L$. Dashed lines are quadratic fits that illustrate the finite condensate in the ordered phases. The solid (red) line is a fit to the form $y=c_{1} x^{c_{2}}$ (illustrated for $J / Q=0.040$ ), where $c_{2}=z+\eta_{\mathrm{N}}$ is expected at the critical coupling. In fitting to the nine $L$ values for each $J / Q$, we find a minimum in the chi-squared value (per degree of freedom) of 3.1 for $J / Q=0.040$, with $c_{2} \approx 1.35(1)$. For $J / Q=0.038$, the chi-squared value is 3.9 , with $c_{2} \approx 1.37(1)$. All other $J / Q$ produce much larger chisquare (greater than 10).

scaling of these observables provides strong evidence for a continuous $z=1$ transition in the JQ model.

Basis and Sign of Matrix Elements: A priori, it is unclear that SSE simulations of $H_{\mathrm{JQ}}$ are free of the notorious sign-problem: a fluctuating sign in the weights used in the QMC sampling. In the SSE, finding an orthogonal basis in which all off-diagonal matrix elements of the Hamiltonian are non-positive solves the sign-problem. A simple unitary transformation on the $S^{z}$ basis (a $\pi$ rotation about the $z$-axis on one sub-lattice) results in a new basis in which, for $J, Q>0$, all off-diagonal matrix elements of $H_{\mathrm{JQ}}$ are non-positive, allowing sign-problem free simulations (Fig. (1). We note that this non-positivity condition is also the main ingredient in the proof of the Marshall sign theorem, allowing us to infer that the ground state of $H_{\mathrm{JQ}}$ for $J, Q>0$ must be a spin-singlet. As shown below, this singlet state changes from Néel at $Q \ll J$ to VBS at $J \ll Q$.

Numerical Results: Using the SSE QMC, we studied various physical observables in the JQ model on finitesize lattices of linear dimension $L$ (with number of spins $N_{\text {spin }}=L^{2}$ ). Particular attention was paid to the scaling of the spin stiffness $\rho_{s}=\partial^{2} E_{0} / \partial \phi^{2}$ ( $E_{0}$ is the energy and $\phi$ is a twist in the boundary conditions) and the uniform spin susceptibility $\chi_{u}=\left\langle\left(\sum_{i} S_{i}^{z}\right)^{2}\right\rangle / T N_{\text {spin }}$. In the $S^{z}$ basis used here, it is easy to measure the correlation functions $C_{\mathrm{N}}^{z}(\mathbf{r}, \tau)=\left\langle S^{z}(\mathbf{r}, \tau) S^{z}(0,0)\right\rangle$ and $C_{\mathrm{V}}^{z}(\mathbf{r}, \tau)=$ $\left\langle\left[S^{z}(\mathbf{r}, \tau) S^{z}(\mathbf{r}+\hat{\mathbf{x}}, \tau)\right]\left[S^{z}(0,0) S^{z}(\hat{\mathbf{x}}, 0)\right]\right\rangle$. While $C_{\mathrm{N}}^{z}$ is the correlation function of the Néel order parameter, the VBS order is indicated by $C_{\mathrm{V}}^{z}$, which is the correlation function of the composite operator $S^{z}(\mathbf{r}) S^{z}(\mathbf{r}+\hat{\mathbf{x}})$, receiving contribution from both the standard VBS order parameter

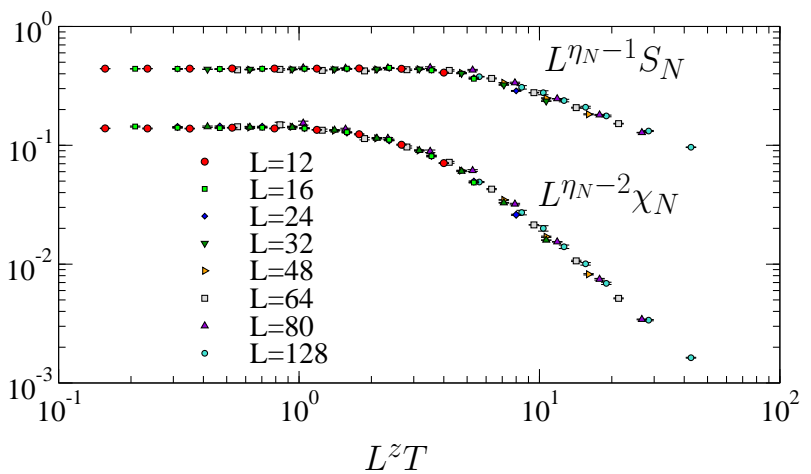

FIG. 3: (color online) Criticality of the Néel field at $J=0.038$ : collapse of the Néel structure factor $\left(S_{N}\right)$ and susceptibility $\left(\chi_{N}\right)$ with $z=1$ and $\eta_{N}=0.35$, determining the universal functions $\mathbb{X}_{\mathrm{S}}(x)$ and $\mathbb{X}_{\chi}(x)$ (up to non-universal scale factors on the $x$ and $y$ axes). The only fit parameter for both $S_{N}$ and $\chi_{N}$ is $\eta_{N}$, the anomalous dimension of the Néel field.

$\mathbf{S}(\mathbf{r}) \cdot \mathbf{S}(\mathbf{r}+\hat{\mathbf{x}})$ as well as the traceless symmetric tensor constructed from $S^{i}(\mathbf{r}) S^{j}(\mathbf{r}+\hat{\mathbf{x}})$. Structure factors for the Néel and VBS phases are constructed from these correlation functions by Fourier transformation at equal time, $S_{\mathrm{N}, \mathrm{V}}[\mathbf{q}]=\sum_{\mathbf{r}}\left[\exp (-i \mathbf{q} \cdot \mathbf{r}) C_{\mathrm{N}, \mathrm{V}}^{z}(\mathbf{r}, \tau=\mathbf{0})\right] / N_{\mathrm{spin}}$, from which the order parameters are defined at the observed ordering wavevectors: $m_{\mathrm{N}, \mathrm{V}}^{2}=S_{\mathrm{N}, \mathrm{V}}\left[\mathbf{q}_{\mathrm{N}, \mathrm{V}}\right] / N_{\text {spin }}$. Zero-frequency susceptibilities $\left(\chi_{\mathrm{N}}\right.$ and $\left.\chi_{\mathrm{V}}\right)$ are constructed by integrating over all $\tau$ and Fourier transforming in space to the ordering vectors, $\mathbf{q}_{\mathrm{N}, \mathrm{V}}$.

Examination of the full $\mathbf{q}$-dependent structure factors indicate the presence of sharp ordering wavevectors in $S_{\mathrm{N}}\left[\mathbf{q}_{\mathrm{N}}=(\pi, \pi)\right]$ for large $J / Q$ and $S_{\mathrm{V}}\left[\mathbf{q}_{\mathrm{V}}=(\pi, 0)\right.$ or $(0, \pi)$ ] (the latter in the case where the correlator is measured with $\hat{\mathbf{y}}$ ) for large $Q / J[8]$, confirming the Néel and VBS phases observed in Ref. [6]. As shown in Fig. 2, $T \rightarrow$ 0 converged data scales convincingly to a non-zero value for $m_{\mathrm{N}}^{2}$ at $J / Q=0.1$ and for $m_{\mathrm{V}}^{2}$ at $J / Q=0.01$. The critical coupling appears to occur between $J_{c} \approx 0.038$ and 0.040 (we set $Q=1$ fixed throughout), such that as $J_{c}$ is approached from above (below) the extrapolated Néel (VBS) order parameter is suppressed. Very near $J_{c}$, both order parameters vanish within our error bars, while a power law with no $y$-intercept fits the Néel data with high accuracy. More specifically, at $J_{c}$, scaling arguments require $S_{N} \propto L^{1-\eta_{N}} \mathbb{X}_{S}\left(L^{z} T / c\right)$ and $\chi_{N} \propto L^{2-\eta_{N}} \mathbb{X}_{\chi}\left(L^{z} T / c\right)$, with $\eta_{N}$ the anomalous dimension of the Néel field. In Fig. 3, we verify this scaling behavior and determine the universal functions $\mathbb{X}_{\mathrm{S}}$ and $\mathbb{X}_{\chi}$. Both analyses illustrated in Figs. (213) give a consistent estimate of $\eta_{N} \approx 0.35(3)$. This value is larger than the result of $\eta_{N} \approx 0.26(3)$ from Ref. [ 6 ]. While the exact source of this discrepancy is unclear due to the entirely different methods used to extract the exponents, we note that (a) our analysis does not involve extra fit parameters from the inclusion of sub-leading corrections, and 


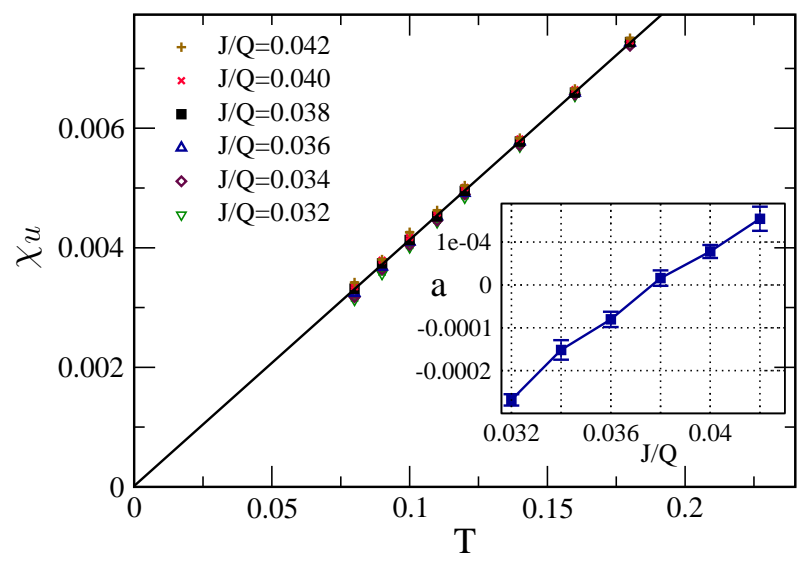

FIG. 4: (color online) Finite- $T$ uniform susceptibility, for a $L=128$ system near $J_{c}$. Error bars are much smaller than the symbol size. For the region $0.08 \leq T \leq 0.18$, the data is highly linear, and a straight-line fit for $J / Q=0.038$ (shown) intercepts the origin within error bars. Intercepts of straightline fits for all data sets are in the inset. From the slope of the linear- $T$ behavior we obtain $\mathcal{A}_{\chi} / c^{2}=0.0412(2)$.

(b) the collapse of both $S_{N}$ and $\chi_{N}$ takes place over two and a half orders of magnitude of $L T$ with only one common fit parameter, $\eta_{N}$; both facts give us confidence in our estimate. The critical scaling of $C_{\mathrm{V}}^{z}$ is more complicated; due to the aforementioned mixing-in of two order parameters, $C_{\mathrm{V}}^{z}$ is expected to receive two independent power-law contributions. Indeed, it is difficult to disentangle these individual contributions on the limited range of lattices sizes available, precluding us from verifying the proposal [6] that $\eta_{\mathrm{N}}=\eta_{\mathrm{V}}$.

We now turn to an analysis of the scaling properties of $\chi_{u}$ and $\rho_{s}$ in the hypothesized quantum critical fan region of Fig $1 \chi_{u}$ and $\rho_{s}$, being susceptibilities of conserved quantities have no anomalous scaling dimension, and hence at finite- $T$ and $L$ in the proximity of a scaleinvariant critical point, assuming hyper-scaling:

$$
\begin{aligned}
\rho_{s}(T, L, J) & =\frac{T}{L^{d-2}} \mathbb{Y}\left(\frac{L^{z} T}{c}, g L^{1 / \nu}\right), \\
\chi_{u}(T, L, J) & =\frac{1}{T L^{d}} \mathbb{Z}\left(\frac{L^{z} T}{c}, g L^{1 / \nu}\right),
\end{aligned}
$$

where $g \propto\left(J-J_{c}\right) / J_{c}$. At criticality $(g=0)$, it is easy to see that $\mathbb{Y}(x \rightarrow 0,0)=\mathcal{A}_{\rho} / x$ and $\mathbb{Z}(x \rightarrow \infty, 0)=$ $\mathcal{A}_{\chi} x^{d / z}$, where $\mathbb{Y}(x, y)$ and $\mathbb{Z}(x, y)$ are universal scaling functions and $\mathcal{A}_{\chi}, \mathcal{A}_{\rho}$ are universal amplitudes of the quantum critical point; $c$ is a non-universal velocity.

At criticality and $L \rightarrow \infty$, one can show from Eq. (3) that $\chi_{u}=\frac{\mathcal{A}_{\chi}}{c^{d / z}} T^{d / z-1}$; i.e. for a $z=1$ transition, $\chi_{u}$ should be $T$-linear and have a zero intercept on the $\mathrm{y}$ axis at $T=0$ [10]. In Fig. 4, $\chi_{u}$ data for an $L=128$ system is presented. Within our error bars, this data is $L \rightarrow \infty$ converged for the region of $T$ shown; at smaller $T$ the finite-size gap causes an exponential reduction in

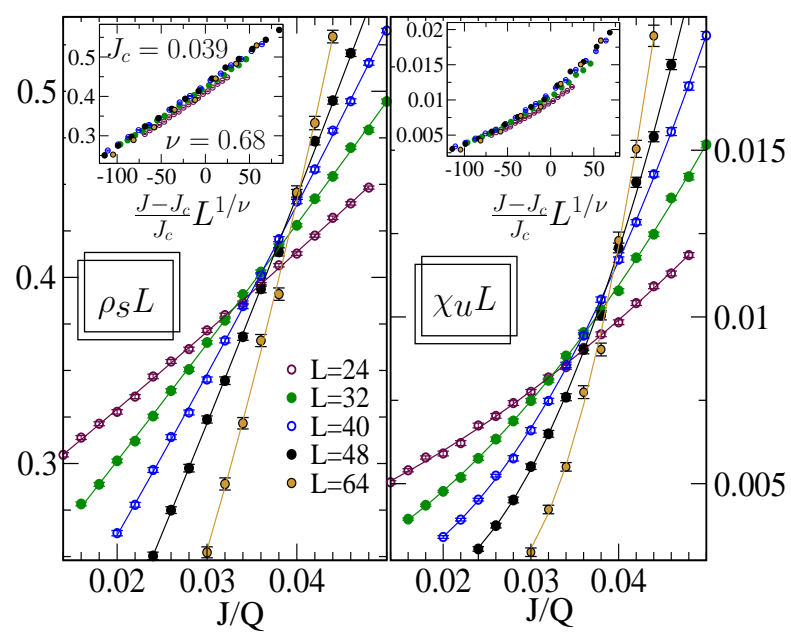

FIG. 5: (color online) Zoom-in of the spin stiffness and susceptibility close to the expected critical point, taken for simulations cells of size $L=1 / T$. Data in the inset is scaled to get the best collapse for the largest system sizes, which occurs for $J_{c} \sim 0.039(1)$ and $\nu \sim 0.68(4)$.

$\chi_{u}$. The inset shows how the extracted value of the $\mathrm{y}$ intercept, $a$ (from a fit to the form $a+b T$ ), changes sign as the coupling is tuned, consistent with $0.036 \leq J_{c} \leq 0.040$ and demonstrating to high precision the $z=1$ scaling.

Turning to study Eqs. (23) further, one may hold the first argument of the universal functions fixed by setting $L=1 / T$ (assuming $z=1$ as indicated above). In order to achieve this, we performed extensive simulations on lattices sizes up to $L=1 / T=64$, illustrated in Fig. 5. According to Eqs. (213), data curves for $L \rho_{s}$ and $L \chi_{u}$ plotted versus $J$ should show a crossing point with different $L$ precisely at $J_{c}$. We find that for relatively large sizes $(32 \leq L \leq 64)$ the crossing point converges quickly in the interval $0.038 \leq J \leq 0.040$. The insets show the data collapse when the $x$-axis is re-scaled to $g L^{1 / \nu}$ (with $\nu=0.68)$. We note that with the inclusion of small sub-leading corrections (of the form $a_{\omega} / L^{\omega}$ ), the crossing point and data collapse of $\rho_{s}$ and $\chi_{u}$ can be made consistent, at the expense of two more fit parameters, even for much smaller system sizes than illustrated [9]. In contrast to the U(1) symmetric JK model [11], where the absence of a $T$-linear $\chi_{u}$ and a crossing in the data for $\rho_{s} L$ cast doubt on its interpretation as a $z=1 \mathrm{QCP}$, the present data for this $\mathrm{SU}(2)$ symmetric model gives strong support for a $z=1 \mathrm{QCP}$ between $0.038 \leq J \leq 0.040$.

Finally, we hold the second argument of the scaling functions [Eqs. (223)] constant by tuning the system to $g=0$. One then expects a data collapse for $\rho_{s} / T$ and $L \chi_{u}$ when they are plotted as a function of $L^{z} T$ (with $z=$ 1). Fig. 6] shows this collapse for simulations carried out with extremely anisotropic arguments $L T$, varying over almost three orders of magnitude. There is an excellent data collapse over 8 orders of magnitude of the range of 


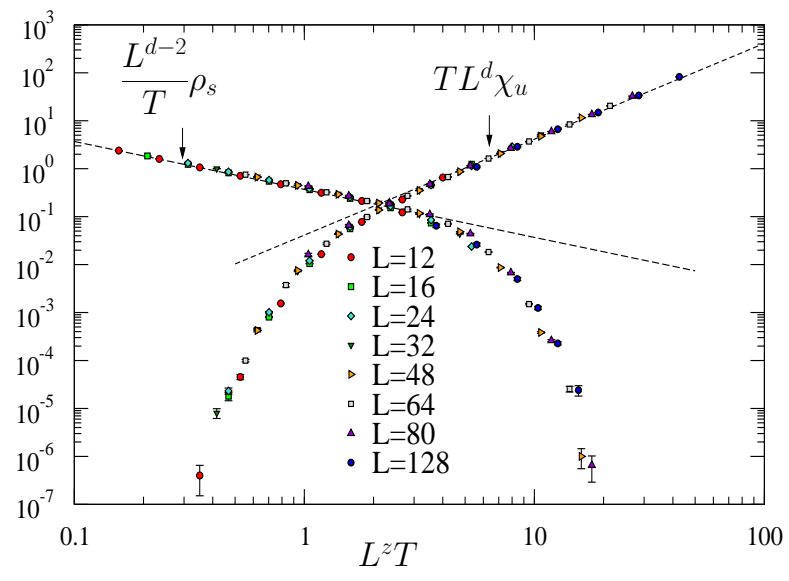

FIG. 6: Scaling of $\chi_{u}$ and $\rho_{s}$ at $J=0.038 \approx J_{c}$, with $z=1$ and $d=2$. These plots are the universal functions $\mathbb{Y}(x, 0)$ and $\mathbb{Z}(x, 0)$ up to the non-universal scale factor $c$ on the $x$-axis. The expected asymptotes (see text) are plotted as dashed lines $\mathbb{Y}(x \rightarrow 0,0)=\mathcal{A}_{\rho} / x$ and $\mathbb{Z}(x \rightarrow \infty, 0)=\mathcal{A}_{\chi} x^{d / z}$. From fits to the data, we find $\mathcal{A}_{\chi} / c^{2}=0.041(4)$ and $\mathcal{A}_{\rho} c=0.37(3)$, allowing us to estimate a universal model-independent number associated with the QCP, $\mathcal{A}_{\rho} \sqrt{\mathcal{A}_{\chi}} \approx 0.075(4)$.

the universal functions, with no fit parameters. This data together with that in Fig. 4 provide our most striking evidence for the existence of a QCP with $z=1$ in the proximity of $J / Q \approx 0.038$.

Discussion: In this paper we have presented extensive data for the $\mathrm{SU}(2)$ symmetric JQ model which indicates that the Néel order (present when $J \gg Q$ ) is destroyed at a continuous quantum transition as $Q$ is increased [6]. In the finite- $T$ quantum critical fan above this QCP, scaling behavior is found that confirms the dynamic scaling exponent $z=1$ to high accuracy. The anomalous dimension of the Néel field at this transition is determined to be $\eta_{\mathrm{N}} \approx 0.35(3)$, almost an order of magnitude more than its value of 0.038 [12] for a conventional $O(3)$ transition. For sufficiently large values of $Q$ we find that the system enters a spin-gapped phase with VBS order. To the accuracy of our simulations, our results are fully consistent with a direct continuous QCP between the Néel and VBS phases, with a critical coupling between $J / Q \approx 0.038$ and $J / Q \approx 0.040$. Although our finite size study cannot categorically rule out a weak first-order transition, we have found no evidence for double-peaked distributions, indicating an absence of this sort of first-order behavior on the relatively large length scales studied here. It is interesting to compare our results to the only theory currently available for a continuous Néel-VBS transition: the deconfined quantum criticality scenario [4], in which the Néel-VBS transition is described by the noncompact $\mathbb{C P}^{1}$ field theory. All of the qualitative observations above, including an unusually large $\eta_{\mathrm{N}}$ [13] agree with the predictions of this theory. Indeed, our estimate of $\eta_{\mathrm{N}} \approx 0.35$ [Fig. 3 is in remarkable numerical agreement with a recent field-theoretic computation 14] of this quantity, which finds $\eta_{\mathrm{N}}=0.3381$. With regard to other detailed quantitative comparisons, we have provided the first step by computing many universal quantities, $\mathbb{X}_{\chi}(x), \mathbb{X}_{S}(x), \mathbb{Y}(x, 0), \mathbb{Z}(x, 0)$ and $\mathcal{A}_{\rho} \sqrt{\mathcal{A}_{\chi}} \approx 0.075$ [Fig. 6] in the JQ model. Analogous computations in the $\mathbb{C P}^{1}$ model, although currently unavailable [15] are highly desirable to further demonstrate that the JQ model realizes this new and exotic class of quantum criticality.

We acknowledge scintillating discussions with S. Chandrasekharan, A. del Maestro, T. Senthil, and especially S. Sachdev and A. Sandvik. This research (RGM) was sponsored by D.O.E. contract DE-AC05-00OR22725. RKK acknowledges financial support from NSF DMR0132874, DMR-0541988 and DMR-0537077. Computing resources were contributed by NERSC (D.O.E. contract DE-AC02-05CH11231), NCCS, the HYDRA cluster at Waterloo, and the DEAS and NNIN clusters at Harvard.

[1] E. Manousakis, Rev. Mod. Phys. 63, 1 (1991).

[2] F. D. M. Haldane, Phys. Rev. Lett. 61, 1029 (1988)

[3] N. Read and S. Sachdev, Phys. Rev. B 42, 4568 (1990).

[4] T. Senthil et al. Science 303, 1490 (2004); Phys. Rev. B 70, 144407 (2004).

[5] S. Sachdev, Quantum Phase Transitions (Cambridge University Press, New York, 1999).

[6] A. W. Sandvik, Phys. Rev. Lett. 98, 227202 (2007).

[7] O. F. Syljuåsen and A. W. Sandvik, Phys. Rev. E 66, 046701 (2002); R. G. Melko and A. W. Sandvik, Phys. Rev. E 72, 026702 (2005).

[8] The VBS order is also visible in measurements of correlation functions between off-diagonal terms [9].

[9] R. K. Kaul and R. G. Melko, unpublished.

[10] A. V. Chubukov et al., Phys. Rev. B 49, 11919 (1994).

[11] A. W. Sandvik and R. G. Melko, cond-mat/0604451 (2006); Ann. Phys. (NY), 321, 1651 (2006).

[12] M. Campostrini et al., Phys. Rev. B 65, 144520 (2002).

[13] O. I. Motrunich and A. Vishwanath, Phys. Rev. B 70, 075104 (2004).

[14] Z. Nazario and D. I. Santiago, Nucl. Phy. B 761, 109 (2007)

[15] A. Kuklov et al., Annals of Physics 321, 1602 (2006); found a discontinuous transition in a $\mathrm{U}(1)$ deformation of the $\mathbb{C P}^{1}$ model. Results relevant to the SU(2) invariant $H_{\mathrm{JQ}}$ are so far unavailable (see however [13]). 